\title{
Flowshop scheduling problem to minimize total completion time with random and bounded processing times
}

\author{
YN Sotskov ${ }^{1}$, A Allahverdi ${ }^{2 *}$ and T-C Lai ${ }^{3}$ \\ ${ }^{1}$ United Institute of Informatics Problems, Minsk, Belarus; ${ }^{2}$ Kuwait University, Safat, Kuwait; ${ }^{3}$ National Taiwan \\ University, Tapei, Taiwan
}

\begin{abstract}
The flowshop scheduling problems with $n$ jobs processed on two or three machines, and with two jobs processed on $k$ machines are addressed where jobs have random and bounded processing times. The probability distributions of random processing times are unknown, and only the lower and upper bounds of processing times are given before scheduling. In such cases, there may not exist a unique schedule that remains optimal for all feasible realizations of the processing times, and therefore, a set of schedules has to be considered which dominates all other schedules for the given criterion. We obtain sufficient conditions when transposition of two jobs minimizes total completion time for the cases of two and three machines. The geometrical approach is utilized for flowshop problem with two jobs and $k$ machines. Journal of the Operational Research Society (2004) 55, 277-286. doi:10.1057/palgrave.jors.2601682
\end{abstract}

Keywords: scheduling; flowshop; uncertainty; geometrical approach; total completion time

\section{Introduction}

The deterministic (where processing times are known with certainty) $k$-machine flowshop scheduling problem with the minimization of total completion time has been considered for both $k=2$ and $k>2$ cases. Gonzalez and Sahni ${ }^{1}$ proved that this problem is unary NP-hard even for the twomachine case $(k=2)$. Therefore, the main research has been focused on the development of implicit enumeration techniques and heuristics. Many researchers considered this problem for $k=2$, see Della Croce et $a l^{2}{ }^{2}$ and Allahverdi. ${ }^{3}$ For $k>2$, many heuristics have been proposed including Rajendran and Ziegler, ${ }^{4}$ Woo and Yim, ${ }^{5}$ and Allahverdi and Aldowaisan. ${ }^{6}$ The two-machine flowshop problem has also been considered for stochastic environments, and the following two types of stochastic problems have been addressed in the OR literature. In a stochastic job problem, processing times are assumed to be random variables following certain probability distributions: mainly the exponential probability distribution has been considered. The stochastic job problem has been addressed for minimization of makespan, see Kamburowski, ${ }^{7}$ and Elmaghraby and Thoney. ${ }^{8}$ In a stochastic machine problem, processing times are usually deterministic (and fixed before scheduling), while job completion times are random variables as a result of possible machine breakdowns. Allahverdi, ${ }^{9}$ and

\footnotetext{
*Correspondence: A Allahverdi, Department of Industrial and Management Systems Engineering, College of Engineering and Petroleum, Kuwait University, PO Box 5969, Safat 13060, Kuwait.

E-mail: allahverdi@kuc01.kuniv.edu.kw
}

Allahverdi and Mittenthal ${ }^{10}$ addressed the stochastic machine problem with total completion time criterion.

In this paper, we address the stochastic job problem and consider a frequent situation when it is hard to obtain exact probability distributions for random processing times and assuming a specific probability distribution (eg, exponential) is not realistic. As such, a solution obtained by assuming a certain probability distribution may not be close to the optimal solution for the realization of the process. It has been observed that although the exact probability distributions of processing times may not be known before scheduling, upper and lower bounds on processing times are easy to obtain in many practical cases.

The considered problem is to minimize total completion time of $n$ jobs $j \in J=\{1,2, \ldots, n\}$ processed on $k$ machines $m \in M=\{1,2, \ldots, k\}$ when only a lower bound $a_{j, m} \geqslant 0$ and an upper bound $b_{j, m} \geqslant a_{j, m}$ of the processing time $t_{j, m}$ of job $j$ on machine $m$ are given before scheduling. All $n$ jobs have the same technological route through $k$ machines, namely, 1 , $2, \ldots, k$. Using the three-field notation of Lawler et al, ${ }^{11}$ this problem may be denoted as $F k\left|a_{j, m} \leqslant t_{j, m} \leqslant b_{j, m}\right| \sum C_{i, k}$. Hereafter, $C_{i, k}$ means the completion time of the job in position $i$ on machine $k$ (the last machine in the technological route), and the criterion $\sum C_{i, k}$ means minimization of total completion time $\sum_{i=1}^{n} C_{i, k}$. For the research on the deterministic problem see Lawler et $a l^{11}$ and Tanaev et al. ${ }^{12}$

It should be noted that random processing times in the problem $F k\left|a_{j, m} \leqslant t_{j, m} \leqslant b_{j, m}\right| \sum C_{i, k}$ are due to external forces in contrast to scheduling problems with controllable processing times. In the latter problem, the objective is to choose 
both optimal processing times (which are under the control of a decision-maker), and an optimal schedule with chosen processing times. ${ }^{13-15}$ Another related yet different problem is so-called hoist scheduling. ${ }^{16,17}$ Hoist scheduling is considered in chemical and electroplating industries, where the objective is to minimize the cycle time of a repetitive process. The hoist has to travel from one tank to another one and lift or drop the job at the suitable time. Because of the nature of the chemical process, the processing time inside each tank has to be controlled within the given lower and upper bounds. In hoist scheduling, the processing times are under the control of decision-maker and are a part of the solution.

The scheduling problems with random and bounded processing times were considered in Lai et al, ${ }^{18}$ and Lai and Sotskov ${ }^{19}$ for jobshop with makespan criterion and in Lai et $a l^{20}$ for jobshop with total completion time criterion. In particular, Lai et $a l^{18}$ provided the formula for calculating the stability radius of the schedule minimizing the makespan, that is, the largest independent variations of the processing times that this schedule remains optimal without fail. In Lai et $a l^{20}$ stability analysis of a schedule minimizing total completion time was involved in a branch-and-bound algorithm for solving jobshop problem $J \mid a_{j, m} \leqslant t_{j, m}$ $\leqslant b_{j, m} \mid \sum C_{i}$.

For a two-machine flowshop problem $F 2 \mid a_{j, m} \leqslant t_{j, m}$ $\leqslant b_{j, m} \mid C_{\max }$, Allahverdi and Sotskov ${ }^{21}$ found sufficient conditions when transposition of two jobs minimizes makespan. In this paper, we address the same problem as Allahverdi and Sotskov ${ }^{21}$ but with total completion time criterion rather than makespan criterion. We also address the three-machine problem with total completion time criterion. Moreover, we consider the two-job $k$-machine problem for any regular objective function. In contrast to Lai et al, ${ }^{18}$ Lai and Sotskov ${ }^{19}$ and Lai et al, ${ }^{20}$ where only exponential algorithms have been derived, in this paper, we develop polynomial procedures for solving special cases of problem $F k\left|a_{j, m} \leqslant t_{j, m} \leqslant b_{j, m}\right| \sum C_{i, k}$. The next section contains main notations and definitions. Dominance relations for flowshop with two and three machines are derived in the subsequent two sections. In the three sections following, we utilize geometrical algorithm for the case of two jobs. Concluding remarks are made in the last section.

\section{Definitions and notations}

Let $t_{j, m}$ denote processing time of operation $O_{j, m}$ of job $j \in J$ processed on machine $m \in M$. It is assumed that pre-emption of operation $O_{j, m}$ is not allowed. Although we do not know the exact value of $t_{j, m}$ before scheduling, we know the lower and upper bounds of feasible processing times $t_{j, m}$ given by inequalities

$$
a_{j, m} \leqslant t_{j, m} \leqslant b_{j, m}
$$

Inequalities (1) define the set (polytope) $T$ of feasible vectors $t=\left(t_{1,1}, t_{1,2}, \ldots, t_{n, k}\right)$ of the processing times: $T=$ $\left\{t: a_{j, m} \leqslant t_{j, m} \leqslant b_{j, m}, j \in J, m \in M\right\}$. For a fixed sequence $\pi=$ $\left(j_{1}, j_{2}, \ldots, j_{n}\right)$ of $n$ jobs, we use the notation $t_{[i, m]}(\pi)$ to denote the processing time of operation $O_{j_{i}, m}$ of job $j_{i} \in J$ which is located in position $i, 1 \leqslant i \leqslant n$, of the sequence $\pi$ on machine $m \in M$. Similar to $t_{j, m}$, the exact value $t_{[i, m]}(\pi)$ is unknown before scheduling, and so the notations $a_{[i, m]}(\pi)$ and $b_{[i, m]}(\pi)$ are used for the lower and upper bounds of processing time $t_{[i, m]}(\pi)$.

Let $\mathbf{S}=\left\{\pi_{1}, \pi_{2}, \ldots, \pi_{n !}\right\}$ be a set of all permutations (sequences) of $n$ jobs from set $J$. The set $\mathbf{S}$ of permutation schedules is dominant for the problem of two-machine flowshop with random processing times. ${ }^{22}$ Since the processing times are random variables for the problem under consideration, permutation schedules are also dominant for the problem $F 2\left|a_{j, m} \leqslant t_{j, m} \leqslant b_{j, m}\right| \sum C_{i, 2}$. However, the set of permutation schedules is not necessarily dominant for the problem of three-machine flowshop, and for the problem with $k \geqslant 3$ and $n=2 .{ }^{23}$ Therefore, for the problem with two jobs and $k \geqslant 3$ machines, we are forced to consider the whole set of active schedules. ${ }^{12}$ using geometrical approach. For the three-machine case, we will consider permutation schedules since such a restriction of feasible schedules considered is commonly used both in theory and in practice.

Let $\pi_{r}(t)$ denote the schedule defined by permutation (sequence) $\pi_{r} \in \mathbf{S}$ for the fixed vector $t \in T$ of processing times and let $C_{i, m}\left(\pi_{\mathrm{r}}(t)\right)$ denote the completion time of job $j_{i}$ in position $i$ on machine $m$ in the schedule $\pi_{r}(t)$.

Definition 1 A set of sequences $\mathbf{S}^{*} \subseteq \mathbf{S}$ is a solution to the problem $F k\left|a_{j, m} \leqslant t_{j, m} \leqslant b_{j, m}\right| \sum C_{i, k}$ if for each feasible vector $t \in T$ of processing times, the set $\mathbf{S}^{*}$ contains at least one sequence $\pi_{r}$ such that schedule $\pi_{r}(t)$ is optimal.

The whole set $\mathbf{S}$ of sequences is an obvious solution to the problem $F k\left|a_{j, m} \leqslant t_{j, m} \leqslant b_{j, m}\right| \sum C_{i, k}$. However if $n$ is large, it is hard and practically infeasible for a scheduler to choose the best sequence from a huge set $\mathbf{S}^{*}=\mathbf{S}$ of candidates as the processing of jobs evolves and additional information about processing times becomes available. Therefore, it is important to minimize the cardinality of set $\mathbf{S}^{*}$ which is a solution to the problem $F k\left|a_{j, m} \leqslant t_{j, m} \leqslant b_{j, m}\right| \sum C_{i, k}$. The desirable case is to construct a solution $\mathbf{S}^{*}$ with minimal cardinality. The following dominance relation is used throughout the paper.

Definition 2 Sequence $\pi_{r} \in \mathbf{S}$ dominates sequence $\pi_{u} \in \mathbf{S}$ with respect to $T$ if the inequality $\sum C_{i, k}\left(\pi_{r}(t)\right) \leqslant \sum C_{i, k}\left(\pi_{u}(t)\right)$ holds for any feasible vector $t \in T$ of processing times.

Let $s_{d}$ with $d \in\{1,2\}$ denote a part (subsequence) of sequence $\pi \in \mathbf{S}$, for example, $\pi=\left(s_{1}, j, s_{2}\right)$ means a sequence of $n$ jobs in which job $j$ is surrounded by subsequences $s_{1}$ and $s_{2}$. If there are some conditions of the form $t_{v, x} \geqslant t_{w, z}$ which are sufficient for the sequence $\pi_{1}=\left(s_{1}, w, v, s_{2}\right) \in \mathbf{S}$ to dominate the sequence $\pi_{2}=\left(s_{1}, v, w, s_{2}\right) \in \mathbf{S}$ with respect to $T=\{t\}$ (ie, for the problem $F k \| \sum C_{i, k}$ ), then we can obtain 
similar sufficient conditions for the problem $F k\left|a_{j, m} \leqslant t_{j, m} \leqslant b_{j, m}\right| \sum C_{i, k}$ by replacing $t_{v, x}$ by $b_{v, x}$ and $t_{w, z}$ by $a_{w, z}$. More complicated sufficient conditions are derived in the following two sections.

\section{Two machines}

Let $T_{j, m}(\pi(t))$ denote the sum of processing times of the jobs in positions $1,2, \ldots, j$ of the sequence $\pi \in \mathbf{S}$ on machine $m \in M$. For brevity, we omit $t$ in this notation: $T_{j, m}(\pi)=\sum_{i=1}^{j} t_{[i, m]}(\pi), j \in\{1,2, \ldots, n\}, m \in\{1,2, \ldots, k\}$. Also let

$$
\begin{aligned}
& \delta_{j}(\pi)=T_{j, 1}(\pi)-T_{j-1,2}(\pi), \quad j \in\{1,2, \ldots, n\}, \\
& \Delta_{j}(\pi)=\max \left\{\delta_{1}(\pi), \delta_{2}(\pi), \ldots, \delta_{j}(\pi)\right\}
\end{aligned}
$$

where the equality $T_{0,2}(\pi)=0$ is assumed. Obviously, $\Delta_{j}(\pi)$ is the total idle time on the second machine until the job in position $j$ of sequence $\pi$ is completed. Observe that

$$
\Delta_{j}(\pi)=\max \left\{\Delta_{j-1}(\pi), \delta_{j}(\pi)\right\}
$$

If $k=2$, the completion time of the job in position $j$ of sequence $\pi$ is given by

$$
C_{j, 2}(\pi)=T_{j, 2}(\pi)+\Delta_{j}(\pi),
$$

and the total completion time for the schedule defined by sequence $\pi$ (for brevity, $\mathrm{TCT}(\pi)$ ) can be calculated as follows:

$$
\operatorname{TCT}(\pi)=\sum_{j=1}^{\mathrm{n}} C_{j, 2}(\pi)=\sum_{j=1}^{n} T_{j, 2}(\pi)+\sum_{j=1}^{n} \Delta_{j}(\pi)
$$

Theorem 1 For the problem $F 2\left|a_{j, m} \leqslant t_{j, m} \leqslant b_{j, m}\right| \sum C_{i, 2}$, the sequence $\pi_{1}=\left(s_{1}, w, v, s_{2}\right) \in \mathbf{S}$ dominates the sequence $\pi_{2}=\left(s_{1}\right.$, $\left.v, w, s_{2}\right) \in \mathbf{S}$ with respect to $T$ if the following three inequalities are satisfied: $a_{v, 2} \geqslant b_{w, 2}, a_{v, 1} \geqslant b_{w, 1}$, and $a_{w, 2} \geqslant \min \left\{b_{v, 1}, b_{w, 1}\right\}$.

Proof Let sequence $\pi_{1}$ have job $w$ in position $\tau$ and job $v$ in position $\tau+1$, and sequence $\pi_{2}$ have job $v$ in position $\tau$ and job $w$ in position $\tau+1$. Since both sequences $\pi_{1}$ and $\pi_{2}$ have the same jobs in positions $1,2, \ldots, \tau-1$ we get the following equations:

$$
\delta_{j}\left(\pi_{1}\right)=\delta_{j}\left(\pi_{2}\right), j=1,2, \ldots, \tau-1
$$

It can easily be shown that

$$
\delta_{j}\left(\pi_{1}\right)=\delta_{j}\left(\pi_{2}\right), j=\tau+2, \tau+3, \ldots, n
$$

It follows from Equation (2) that $\delta_{\tau}\left(\pi_{1}\right)=T_{\tau-1,1}\left(\pi_{1}\right)+$ $t_{w, 1}-T_{\tau-1,2}\left(\pi_{1}\right), \quad \delta_{\tau}\left(\pi_{2}\right)=T_{\tau-1,1}\left(\pi_{2}\right)+t_{v, 1}-T_{\tau-1,2}\left(\pi_{2}\right), \quad$ and $\delta_{\tau+1}\left(\pi_{1}\right)=T_{\tau-1,1}\left(\pi_{1}\right)+t_{w, 1}+t_{v, 1}-T_{\tau-1,2}\left(\pi_{1}\right)-t_{w, 2} \quad$ where
$T_{\tau-1,1}\left(\pi_{1}\right)=T_{\tau-1,1}\left(\pi_{2}\right)$ and $T_{\tau-1,2}\left(\pi_{1}\right)=T_{\tau-1,2}\left(\pi_{2}\right)$ since both sequences $\pi_{1}$ and $\pi_{2}$ have the same jobs in positions $1,2, \ldots$, $\tau-1$. From the latter five equations, we obtain

$$
\begin{gathered}
\delta_{\tau}\left(\pi_{1}\right)-\delta_{\tau}\left(\pi_{2}\right)=t_{w, 1}-t_{v, 1} \\
\delta_{\tau+1}\left(\pi_{1}\right)-\delta_{\tau}\left(\pi_{1}\right)=t_{v, 1}-t_{w, 2} \\
\delta_{\tau+1}\left(\pi_{1}\right)-\delta_{\tau}\left(\pi_{2}\right)=\tau_{w, 1}-\tau_{w, 2}
\end{gathered}
$$

It follows from Equation (7) and the hypothesis $a_{v, 1} \geqslant b_{w, 1}$ that

$$
\delta_{\tau}\left(\pi_{1}\right) \leqslant \delta_{\tau}\left(\pi_{2}\right)
$$

regardless what feasible values $t_{v, 1}$ and $t_{w, 1}$ assume. Therefore, from Equation (5) and Inequality (10) we obtain $\max \left\{\Delta_{\tau-1}\left(\pi_{1}\right), \delta_{\tau}\left(\pi_{1}\right)\right\} \leqslant \max \left\{\Delta_{\tau-1}\left(\pi_{2}\right), \delta_{\tau}\left(\pi_{2}\right)\right\}$. Owing to (3), the later inequality is equivalent to

$$
\Delta_{\tau}\left(\pi_{1}\right) \leqslant \Delta_{\tau}\left(\pi_{2}\right)
$$

From the condition $a_{w, 2} \geqslant \min \left\{b_{v, 1}, b_{w, 1}\right\}$ of the theorem, it follows that either $a_{w, 2} \geqslant b_{v, 1}$ or $a_{w, 2} \geqslant b_{w, 1}$. If $a_{w, 2} \geqslant b_{v, 1}$, then due to (8) we get

$$
\delta_{\tau+1}\left(\pi_{1}\right) \leqslant \delta_{\tau}\left(\pi_{1}\right)
$$

If $a_{w, 2} \geqslant b_{w, 1}$, then due to (9) we get

$$
\delta_{\tau+1}\left(\pi_{1}\right) \leqslant \delta_{\tau}\left(\pi_{2}\right)
$$

Therefore, if either (12) or (13) holds, then due to (5) and (10) we get

$$
\begin{aligned}
& \max \left\{\Delta_{\tau-1}\left(\pi_{1}\right), \delta_{\tau}\left(\pi_{1}\right), \delta_{\tau+1}\left(\pi_{1}\right)\right\} \\
& \leqslant \max \left\{\Delta_{\tau-1}\left(\pi_{2}\right), \delta_{\tau}\left(\pi_{2}\right), \delta_{\tau+1}\left(\pi_{2}\right)\right\}
\end{aligned}
$$

Since both sequences $\pi_{1}$ and $\pi_{2}$ have the same jobs in positions $1,2, \ldots, \tau-1$, we get

$$
C_{j, 2}\left(\pi_{1}\right)=C_{j, 2}\left(\pi_{2}\right), j=1,2, \ldots, \tau-1
$$

From Equalities (3), (4), (6) and Inequality (11), it can be shown that

$$
C_{j, 2}\left(\pi_{1}\right) \leqslant C_{j, 2}\left(\pi_{2}\right), \quad j=\tau+2, \tau+3, \ldots, n
$$

Taking the difference between the completion times of the jobs in positions $\tau$ and $\tau+1$ in sequences $\pi_{1}$ and $\pi_{2}$ yields

$$
\begin{aligned}
& C_{\tau, 2}\left(\pi_{1}\right)+C_{\tau+1,2}\left(\pi_{1}\right)-C_{\tau, 2}\left(\pi_{2}\right)-C_{\tau+1,2}\left(\pi_{2}\right)=t_{w, 2} \\
& -t_{v, 2}+\max \left\{\Delta_{\tau-1}\left(\pi_{1}\right), \delta_{\tau}\left(\pi_{1}\right)\right\} \\
& -\max \left\{\Delta_{\tau-1}\left(\pi_{2}\right), \delta_{\tau}\left(\pi_{2}\right)\right\} \\
& +\max \left\{\Delta_{\tau-1}\left(\pi_{1}\right), \delta_{\tau}\left(\pi_{1}\right), \delta_{\tau+1}\left(\pi_{1}\right)\right\} \\
& -\max \left\{\Delta_{\tau-1}\left(\pi_{2}\right), \delta_{\tau}\left(\pi_{2}\right), \delta_{\tau+1}\left(\pi_{2}\right)\right\}
\end{aligned}
$$


By the hypothesis $a_{v, 2} \geqslant b_{w, 2}$ and Inequalities (11) and (14), we obtain

$$
C_{\tau, 2}\left(\pi_{1}\right)+C_{\tau+1,2}\left(\pi_{1}\right) \leqslant C_{\tau, 2}\left(\pi_{2}\right)+C_{\tau+1,2}\left(\pi_{2}\right)
$$

It follows from Equations (15)-(17) that $\operatorname{TCT}\left(\pi_{1}\right) \leqslant$ $\operatorname{TCT}\left(\pi_{2}\right)$ which concludes the proof.

Obviously, Theorem 1 holds for the case $T=\{t\}$, that is, for the problem $F 2 \| \sum C_{i, 2}$. Thus, the next corollary follows.

Corollary 1 For the problem $F 2 \| \sum C_{i, 2}$, the sequence $\pi_{1}=\left(s_{1}, w, v, s_{2}\right) \in \mathbf{S}$ dominates the sequence $\pi_{2}=\left(s_{1}, v, w\right.$, $\left.s_{2}\right) \in \mathbf{S}$ with respect to $T=\{t\}$ if the following three inequalities are satisfied: $t_{v, 2} \geqslant t_{w, 2}, t_{v, 1} \geqslant t_{w, 1}$, and $t_{w, 2} \geqslant \min \left\{t_{v, 1} t_{w, 1}\right\}$.

Next, we derive a similar dominance relation for the case of three machines. Although the two-machine case can be seen as a particular case of the three-machine case, Theorem 1 is not a particular case of Theorem 2 proven in the following section.

\section{Three machines}

Let $f_{j}(\pi)=\Delta_{j}(\pi)+T_{j, 2}(\pi)-T_{j-1,3}(\pi)$ where $T_{0,3}(\pi)=0$, and let $F_{j}(\pi)=\max \left\{f_{1}(\pi), f_{2}(\pi), \ldots, f_{j}(\pi)\right\}$. It can be shown that

$$
C_{j, 3}(\pi)=T_{j, 3}(\pi)+F_{j}(\pi)
$$

Thus if $k=3$, the total completion time for the schedule defined by sequence $\pi$ can be calculated as follows:

$$
\operatorname{TCT}(\pi)=\sum_{j=1}^{n} C_{j, 3}(\pi)=\sum_{j=1}^{n} T_{j, 3}(\pi)+\sum_{j=1}^{n} F_{j}(\pi)
$$

We consider a sequence $\pi_{1}=\left(s_{1}, w, v, s_{2}\right) \in \mathbf{S}$, which has job $w$ in position $\tau$ and job $v$ in position $\tau+1$, and a sequence $\pi_{2}=\left(s_{1}, v, w, s_{2}\right) \in \mathbf{S}$, which has job $v$ in position $\tau$ and job $w$ in position $\tau+1$.

Lemma 1 Equality $f_{r}\left(\pi_{1}\right)=f_{r}\left(\pi_{2}\right)$ holds for $r=1,2, \ldots, \tau-1$, and if $\max \left\{\Delta_{\tau-1}\left(\pi_{1}\right), \delta_{\tau}\left(\pi_{1}\right), \delta_{\tau+1}\left(\pi_{1}\right)\right\} \leqslant \max \left\{\Delta_{\tau-1}\left(\pi_{2}\right), \delta_{\tau}\left(\pi_{2}\right)\right.$, $\left.\delta_{\tau+1}\left(\pi_{2}\right)\right\}$, then $f_{r}\left(\pi_{1}\right) \leqslant f_{r}\left(\pi_{2}\right)$ for $r=\tau+2, \tau+3, \ldots, n$.

Proof It is clear that $f_{r}\left(\pi_{1}\right)=f_{r}\left(\pi_{2}\right)$ for $r=1,2, \ldots, \tau-1$ since both sequences $\pi_{1}$ and $\pi_{2}$ have the same jobs in the first $\tau-1$ positions. For $r=\tau+2, \tau+3, \ldots, n$ it follows from the definition of $f_{r}(\pi)$ that $f_{r}\left(\pi_{1}\right)-f_{r}\left(\pi_{2}\right)=\max \left\{\delta_{1}\left(\pi_{1}\right), \ldots, \delta_{\tau}\left(\pi_{1}\right)\right.$, $\left.\delta_{\tau+1}\left(\pi_{1}\right), \ldots, \delta_{r}\left(\pi_{1}\right)\right\}-\max \left\{\delta_{1}\left(\pi_{2}\right), \ldots, \delta_{\tau}\left(\pi_{2}\right), \delta_{\tau+1}\left(\pi_{2}\right), \ldots\right.$, $\left.\delta_{\mathrm{r}}\left(\pi_{2}\right)\right\}$.

If $\max \left\{\Delta_{\tau-1}\left(\pi_{1}\right), \delta_{\tau}\left(\pi_{1}\right), \delta_{\tau+1}\left(\pi_{1}\right)\right\} \leqslant \max \left\{\Delta_{\tau-1}\left(\pi_{2}\right), \delta_{\tau}\left(\pi_{2}\right)\right.$, $\left.\delta_{\tau+1}\left(\pi_{2}\right)\right\}$, then due to Equality (6) we get $f_{r}\left(\pi_{1}\right) \leqslant f_{r}\left(\pi_{2}\right)$.

Lemma 2 Equality $C_{r, 3}\left(\pi_{1}\right)=C_{r, 3}\left(\pi_{2}\right)$ holds for $r=1,2, \ldots$, $\tau-1$. Moreover, if $\max \left\{F_{\tau-1}\left(\pi_{1}\right), \quad f_{\tau}\left(\pi_{1}\right)\right.$, $\left.f_{\tau+1}\left(\pi_{1}\right)\right\} \leqslant \max \left\{F_{\tau-1}\left(\pi_{2}\right), f_{\tau}\left(\pi_{2}\right), f_{\tau+1}\left(\pi_{2}\right)\right\}$ and $\max \left\{\Delta_{\tau-1}\left(\pi_{1}\right)\right.$, $\left.\delta_{\tau}\left(\pi_{1}\right), \quad \delta_{\tau+1}\left(\pi_{1}\right)\right\} \leqslant \max \left\{\Delta_{\tau-1}\left(\pi_{2}\right), \quad \delta_{\tau}\left(\pi_{2}\right), \quad \delta_{\tau+1}\left(\pi_{2}\right)\right\}$, then $C_{r, 3}\left(\pi_{1}\right) \leqslant C_{r, 3}\left(\pi_{2}\right)$ for $r=\tau+2, \tau+3, \ldots, n$.

Proof Since both sequences have the same jobs in positions $1,2, \ldots, \tau-1$, we get $C_{r, 3}\left(\pi_{1}\right)=C_{r, 3}\left(\pi_{2}\right)$ for $r=1,2, \ldots, \tau-1$. For $r=\tau+2, \tau+3, \ldots, \quad n$ it follows from (18) that $C_{r, 3}\left(\pi_{1}\right)-C_{r, 3}\left(\pi_{2}\right)=\max \left\{f_{1}\left(\pi_{1}\right), \ldots, \quad f_{\tau}\left(\pi_{1}\right), \quad f_{\tau+1}\left(\pi_{1}\right), \ldots\right.$, $\left.f_{\mathrm{r}}\left(\pi_{1}\right)\right\}-\max \left\{f_{1}\left(\pi_{2}\right), \ldots, f_{\tau}\left(\pi_{2}\right), f_{\tau+1}\left(\pi_{2}\right), \ldots, f_{r}\left(\pi_{2}\right)\right\}$.

If $\max \left\{F_{\tau-1}\left(\pi_{1}\right), f_{\tau}\left(\pi_{1}\right), f_{\tau+1}\left(\pi_{1}\right)\right\} \leqslant \max \left\{F_{\tau-1}\left(\pi_{2}\right), f_{\tau}\left(\pi_{2}\right)\right.$, $\left.f_{\tau+1}\left(\pi_{2}\right)\right\} \quad$ and $\quad \max \left\{\Delta_{\tau-1}\left(\pi_{1}\right), \quad \delta_{\tau}\left(\pi_{1}\right), \quad \delta_{\tau+1}\left(\pi_{1}\right)\right\} \leqslant$ $\max \left\{\Delta_{\tau-1}\left(\pi_{2}\right), \delta_{\tau}\left(\pi_{2}\right), \delta_{\tau+1}\left(\pi_{2}\right)\right\}$, then due to Lemma 1 we get $C_{r, 3}\left(\pi_{1}\right) \leqslant C_{r, 3}\left(\pi_{2}\right)$.

Theorem 2 For the problem $F 3\left|a_{j, m} \leqslant t_{j, m} \leqslant b_{j, m}\right| \sum C_{i, 3}$, the sequence $\pi_{1}=\left(s_{1}, w, v, s_{2}\right) \in \mathbf{S}$ dominates the sequence $\pi_{2}=\left(s_{1}\right.$, $\left.v, w, s_{2}\right) \in \mathbf{S}$ with respect to $T$ if the following four conditions are satisfied:

- $a_{v, 1} \geqslant b_{w, 1}$,

- $a_{v, 2} \geqslant b_{w, 2}$

- $a_{v, 3} \geqslant b_{w, 3}$

- either $\left(a_{w, 3} \geqslant b_{v, 2}\right.$ and $\left.a_{w, 2} \geqslant b_{v, 1}\right)$ or $\left(a_{w, 3} \geqslant b_{w, 2}\right.$ and $\left.a_{w, 2} \geqslant b_{w, 1}\right)$.

Proof We consider the two sequences $\pi_{1}$ and $\pi_{2}$ that have different jobs $v$ and $w$ in positions $\tau$ and $\tau+1$. It follows from the hypothesis $a_{v, 1} \geqslant b_{w, 1}$ and either $a_{w, 2} \geqslant b_{v, 1}$ or $a_{w, 2} \geqslant b_{w, 1}$, and Equations (7)-(9) that $\max \left\{\Delta_{\tau-1}\left(\pi_{1}\right)\right.$, $\left.\delta_{\tau}\left(\pi_{1}\right)\right\} \leqslant \max \left\{\Delta_{\tau-1}\left(\pi_{2}\right), \delta_{\tau}\left(\pi_{2}\right)\right\}$ and

$$
\begin{aligned}
& \max \left\{\Delta_{\tau-1}\left(\pi_{1}\right), \delta_{t}\left(\pi_{1}\right), \delta_{\tau+1}\left(\pi_{1}\right)\right\} \\
& \leqslant \max \left\{\Delta_{\tau-1}\left(\pi_{2}\right), \delta_{\tau}\left(\pi_{2}\right), \delta_{\tau+1}\left(\pi_{2}\right)\right\}
\end{aligned}
$$

Therefore, the condition given in Lemma 1 holds. The $f_{r}(\pi)$ values for the jobs in positions $r=\tau$ and $r=\tau+1$ for the two sequences $\pi=\pi_{1}$ and $\pi=\pi_{2}$ are given as follows:

$$
\begin{aligned}
f_{\tau}\left(\pi_{1}\right)= & T_{\tau-1,2}\left(\pi_{1}\right)+t_{w, 2} \\
& +\max \left\{\Delta_{\tau-1}\left(\pi_{1}\right), \delta_{\tau}\left(\pi_{1}\right)\right\} \\
& -T_{\tau-1,3}\left(\pi_{1}\right) \\
f_{\tau}\left(\pi_{2}\right)= & T_{\tau-1,2}\left(\pi_{2}\right)+t_{v, 2} \\
& +\max \left\{\Delta_{\tau-1}\left(\pi_{2}\right), \delta_{\tau}\left(\pi_{2}\right)\right\} \\
& -T_{\tau-1,3}\left(\pi_{2}\right) \\
& \\
f_{\tau+1}\left(\pi_{1}\right)= & +T_{\tau-1,2}\left(\pi_{1}\right)+t_{w, 2}+t_{v, 2} \\
& +\max \left\{\Delta_{\tau-1}\left(\pi_{1}\right), \delta_{\tau}\left(\pi_{1}\right),\right. \\
& \left.\delta_{\tau+1}\left(\pi_{1}\right)\right\}-T_{\tau-1,3}\left(\pi_{1}\right)-t_{w, 3} \\
& \\
f_{\tau+1}\left(\pi_{2}\right)= & T_{\tau-1,2}\left(\pi_{2}\right)+t_{v, 2}+t_{w, 2} \\
& +\max \left\{\Delta_{\tau-1}\left(\pi_{2}\right), \delta_{\tau}\left(\pi_{2}\right), \delta_{\tau+1}\right. \\
& \left.\left(\pi_{2}\right)\right\}-T_{\tau-1,3}\left(\pi_{2}\right)-t_{v, 3}
\end{aligned}
$$


As long as both sequences have the same jobs in positions 1 through $\tau-1$, we get

$$
\begin{aligned}
T_{\tau-1,2}\left(\pi_{1}\right)= & T_{\tau-1,2}\left(\pi_{2}\right), T_{\tau-1,3}\left(\pi_{1}\right) \\
& =T_{\tau-1,3}\left(\pi_{2}\right), \text { and } \Delta_{\tau-1}\left(\pi_{1}\right) \\
& =\Delta_{\tau-1}\left(\pi_{2}\right)
\end{aligned}
$$

From the above equations, we get

$$
\begin{gathered}
f_{\tau}\left(\pi_{1}\right)-f_{\tau}\left(\pi_{2}\right)=t_{w, 2}-t_{v, 2}+\max \left\{\Delta_{\tau-1}\left(\pi_{1}\right), \delta_{t}\left(\pi_{1}\right)\right\} \\
-\max \left\{\Delta_{t-1}\left(\pi_{2}\right), \delta_{\tau}\left(\pi_{2}\right)\right\} \\
f_{\tau+1}\left(\pi_{1}\right)-f_{\tau}\left(\pi_{1}\right)=t_{v, 2}-t_{w, 3} \\
+\max \left\{\Delta_{\tau-1}\left(\pi_{1}\right), \delta_{\tau}\left(\pi_{1}\right), \delta_{\tau+1}\left(\pi_{1}\right)\right\} \\
-\max \left\{\Delta_{\tau-1}\left(\pi_{1}\right), \delta_{\tau}\left(\pi_{1}\right)\right\} \\
\\
f_{\tau+1}\left(\pi_{1}\right)-f_{\tau}\left(\pi_{2}\right)=t_{w, 2}-t_{w, 3} \\
+\max \left\{\Delta_{\tau-1}\left(\pi_{1}\right), \delta_{\tau}\left(\pi_{1}\right), \delta_{\tau+1}\left(\pi_{1}\right)\right\} \\
-\max \left\{\Delta_{\tau-1}\left(\pi_{2}\right), \delta_{\tau}\left(\pi_{2}\right)\right\}
\end{gathered}
$$

It follows from the conditions $a_{v, 1} \geqslant b_{w, 1}$ and $a_{v, 2} \geqslant b_{w, 2}$ and either $\left(a_{w, 3} \geqslant b_{v, 2}\right.$ and $\left.a_{w, 2} \geqslant b_{v, 1}\right)$ or $\left(a_{w, 3} \geqslant b_{w, 2}\right.$ and $\left.a_{w, 2} \geqslant b_{w, 1}\right)$, and Equalities (20)-(22) that

$$
\begin{gathered}
\max \left\{F_{\tau-1}\left(\pi_{1}\right), f_{\tau}\left(\pi_{1}\right)\right\} \\
\leqslant \max \left\{F_{\tau-1}\left(\pi_{2}\right), f_{\tau}\left(\pi_{2}\right)\right\} \\
\max \left\{F_{\tau-1}\left(\pi_{1}\right), f_{\tau}\left(\pi_{1}\right), f_{\tau+1}\left(\pi_{1}\right)\right\} \\
\leqslant \max \left\{F_{\tau-1}\left(\pi_{2}\right), f_{\tau}\left(\pi_{2}\right), f_{\tau+1}\left(\pi_{2}\right)\right\}
\end{gathered}
$$

Hence, due to Inequalities (19) and (24), the conditions stated in Lemma 2 are satisfied. The completion times of the jobs in positions $\tau$ and $\tau+1$ on the third machine are given as

$$
\begin{gathered}
C_{\tau, 3}\left(\pi_{1}\right)=T_{\tau-1,3}\left(\pi_{1}\right)+t_{w, 3}+\max \left\{F_{\tau-1}\left(\pi_{1}\right), f_{\tau}\left(\pi_{1}\right)\right\} \\
C_{\tau, 3}\left(\pi_{2}\right)=T_{\tau-1,3}\left(\pi_{2}\right)+t_{v, 3}+\max \left\{F_{\tau-1}\left(\pi_{2}\right), f_{\tau}\left(\pi_{2}\right)\right\} \\
C_{\tau+1,3}\left(\pi_{1}\right)=T_{\tau-1,3}\left(\pi_{1}\right)+t_{w, 3}+t_{v, 3} \\
+\max \left\{F_{\tau-1}\left(\pi_{1}\right), f_{\tau}\left(\pi_{1}\right), f_{\tau+1}\left(\pi_{1}\right)\right\} \\
\\
C_{\tau+1,3}\left(\pi_{2}\right)=T_{\tau-1,3}\left(\pi_{2}\right)+t_{v, 3}+t_{w, 3} \\
+\max \left\{F_{\tau-1}\left(\pi_{2}\right), f_{\tau}\left(\pi_{2}\right), f_{\tau+1}\left(\pi_{2}\right)\right\}
\end{gathered}
$$

From the last four equations, we get

$$
\begin{aligned}
& C_{\tau, 3}\left(\pi_{1}\right)+C_{\tau+1,3}\left(\pi_{1}\right)-C_{\tau, 3}\left(\pi_{2}\right)-C_{\tau+1,3}\left(\pi_{2}\right) \\
& =t_{w, 3}-t_{v, 3}+\max \left\{F_{\tau-1}\left(\pi_{1}\right), f_{\tau}\left(\pi_{1}\right)\right\} \\
& -\max \left\{F_{\tau-1}\left(\pi_{2}\right), f_{\tau}\left(\pi_{2}\right)\right\} \\
& +\max \left\{F_{\tau-1}\left(\pi_{1}\right), f_{\tau}\left(\pi_{1}\right), f_{\tau+1}\left(\pi_{1}\right)\right\} \\
& -\max \left\{F_{\tau-1}\left(\pi_{2}\right), f_{\tau}\left(\pi_{2}\right), f_{\tau+1}\left(\pi_{2}\right)\right\}
\end{aligned}
$$

Now it follows from the condition $a_{v, 3} \geqslant b_{w, 3}$ and Inequalities (23) and (24) that

$$
\begin{aligned}
& C_{\tau, 3}\left(\pi_{1}\right)+C_{\tau+1,3}\left(\pi_{1}\right) \\
& \leqslant C_{\tau, 3}\left(\pi_{2}\right)+C_{\tau+1,3}\left(\pi_{2}\right)
\end{aligned}
$$

From Inequality (25) and Lemma 2, we get $\operatorname{TCT}\left(\pi_{1}\right) \leqslant \operatorname{TCT}\left(\pi_{2}\right)$ which concludes the proof.

Since Theorem 2 holds for the case $T=\{t\}$, the next corollary follows.

Corollary 2 For the problem F3\| $\sum C_{i, 3}$, the sequence $\pi_{1}=\left(s_{1}, w, v, s_{2}\right) \in \mathbf{S}$ dominates the sequence $\pi_{2}=\left(s_{1}, v, w\right.$, $\left.s_{2}\right) \in \mathbf{S}$ with respect to $T=\{t\}$ if the following four conditions are satisfied:

- $t_{v, 1} \geqslant t_{w, 1}$,

- $t_{v, 2} \geqslant t_{w, 2}$,

- $t_{v, 3} \geqslant t_{w, 3}$,

- either $\left(t_{w, 3} \geqslant t_{v, 2}\right.$ and $\left.t_{w, 2} \geqslant t_{v, 1}\right)$ or $\left(t_{w, 3} \geqslant t_{w, 2}\right.$ and $\left.t_{w, 2} \geqslant t_{w, 1}\right)$.

Theorems 1 and 2 give sufficient conditions when sequence $\pi_{1}$ dominates sequence $\pi_{2}$. Testing these conditions may restrict solution $\mathbf{S}^{*}$ for problem $F k \mid a_{j, m} \leqslant t_{j, m}$ $\leqslant b_{j, m} \mid \sum C_{i, k}$ with $k=2$ or $k=3$. If after such testing all $n$ jobs will be linearly ordered, then $\left|\mathbf{S}^{*}\right|=1$. In general case when $\left|\mathbf{S}^{*}\right|>1$, one can partition the set $T$ in an appropriate way and then test dominance relations for new problems with smaller sets of feasible vectors of the processing times. Of course, if $T$ is large such an approach may be timeconsuming.

Since, in general, dominance relations do not yield an optimal solution, these relations are often used in an implicit enumeration technique (such as a branch-and-bound algorithm or a dynamic programming) or in a heuristic algorithm. When these relations are used in an implicit enumeration technique, they help in reducing the search space for the optimal solution further. In the case of a heuristic algorithm, the solution is further improved by applying the dominance relations to the solution obtained from the heuristic algorithm.

In the next section, we demonstrate an efficient geometrical approach for the problem with two jobs, $k$ machines and any regular criterion.

\section{Geometrical algorithm for problem $F k|n=2| \Phi$}

Since the set of permutation schedules is not dominant for the considered problem with $k \geqslant 3$ and $n=2$, in this and the next two sections, we consider the whole set of active schedules including the set of permutation schedules as a proper subset. Note that the number of active schedules for problem $F k\left|n=2, a_{j, m} \leqslant t_{j, m} \leqslant b_{j, m}\right| \Phi$ may be equal to $2^{k}$.

For the problem $J|n=2| C_{\max }$, the geometrical algorithm was originally proposed by Akers and Friedman, ${ }^{24}$ and 
developed by Szwarc, ${ }^{25}$ Hardgrave and Nemhauser ${ }^{26}$ and Brucker. ${ }^{27}$ Sotskov ${ }^{28,29}$ generalized the geometrical algorithm for the problem $J k|n=2| \Phi$ with any regular criterion $\Phi$. Next, we describe this algorithm for the problem $F k|n=2| \Phi$.

Let $T M_{j, m}$ denote the sum of processing times of job $j \in J$ on a set of machines $\{1,2, \ldots, \quad m\} \subseteq M$ : $T M_{j, m}=\sum_{i=1}^{m} t_{j, i}, j \in J=\{1,2\}, 1 \leqslant m \leqslant k$. It is assumed that $T M_{1,0}=T M_{2,0}=0$. We introduce a coordinate system on the plane $x, y$ and draw the rectangle $R$ with corners $(0,0)$, $\left(T M_{1, k}, 0\right),\left(0, T M_{2, k}\right),\left(T M_{1, k}, T M_{2, k}\right)$, and rectangles $R_{m}$, $m=1,2, \ldots, k$, with corners $\left(T M_{1, m-1}, T M_{2, m-1}\right),\left(T M_{1, m}\right.$, $\left.T M_{2, m-1}\right),\left(T M_{1, m-1}, T M_{2, m}\right),\left(T M_{1, m}, T M_{2, m}\right)$. For brevity, we call south-west corner $\left(T M_{1, m-1}, T M_{2, m-1}\right)$ of the rectangle $R_{m}$ as $\mathrm{SW}_{m}$, north-west corner $\left(T M_{1, m-1}, T M_{2, m}\right)$ as $\mathrm{NW}_{m}$, south-east corner $\left(T M_{1, m}, T M_{2, m-1}\right)$ as $\mathrm{SE}_{\mathrm{m}}$, and north-east corner $\left(T M_{1, m}, T M_{2, m}\right)$ as $N E_{m}$. The length of a segment connecting points $(x, y)$ and $\left(x^{\prime}, y^{\prime}\right)$ in the rectangle $R$ is defined as $d\left((x, y),\left(x^{\prime}, y^{\prime}\right)\right)=\max \left\{\left|x-x^{\prime}\right|,\left|y-y^{\prime}\right|\right\}$.

An active schedule can be represented on the plane $x, y$ as a trajectory (continuous polygonal line) $\tau=\left[(0,0),\left(x_{1}, y_{1}\right)\right.$, $\left.\left(x_{2}, y_{2}\right), \ldots,\left(x_{r}, y_{r}\right),\left(T M_{1, k}, T M_{2, k}\right)\right]$ where either $x_{r}=T M_{1, k}$ or $y_{r}=T M_{2, k}$. Indeed, let a point $(x, y)$ belong to the trajectory $\tau$ and let $d$ be the length of the trajectory $\tau$ from the point $(0,0)$ to the point $(x, y)$. The coordinate $x$ (coordinate $y$ ) of point $(x, y)$ defines the state of processing job 1 (job 2) as follows. If $\mathrm{SW}_{u}<x \leqslant \mathrm{SE}_{u}$ and $\mathrm{SW}_{v}<y \leqslant \mathrm{NW}_{v}, u \in M, v \in M$, then for the schedule represented by trajectory $\tau$, job 1 (job 2 ) is completed on the machines $1,2, \ldots, u-1$ (on the machines $1,2, \ldots, v-1$ ) before time $d$. Moreover, at time $d$ job 1 (job 2) has been processed on machine $u$ (machine $v$ ) during $x-\mathrm{SW}_{u}$ (during $y-\mathrm{SW}_{v}$ ) time units. Since a machine cannot process more than one job at a time and pre-emption of an operation is forbidden, each straight segment $\left[(x, y),\left(x^{\prime}, y^{\prime}\right)\right]$ of trajectory $\tau$ may be either horizontal (when only job 1 is processed) or vertical (when only job 2 is processed) or diagonal with slope of $45^{\circ}$ (when both jobs 1 and 2 are processed simultaneously). It is clear that a horizontal (vertical) segment can only pass along south or north (west or east) boundary of rectangle $R_{m}, m \in M$, or along north and south (west and east) boundary of rectangle $R$. The diagonal segment of a trajectory $\tau$ can only pass either outside rectangle $R_{m}$ or through point $\mathrm{NW}_{m}$ or point $\mathrm{SE}_{m}$. Problem $F k|n=2| \Phi$ of finding the optimal schedule (trajectory) can be reduced to the shortest path problem in a digraph $(V, A)$ constructed by the following algorithm. Let set $V$ be a subset of set $V^{0}=\left\{(0,0), \quad\left(T M_{1, k}, \quad T M_{2, k}\right)\right\} \cup\left\{\mathrm{NW}_{m}, \quad \mathrm{SE}_{m}: m \in M\right\} \cup$ $\left\{\left(x_{m}, T M_{2, k}\right),\left(T M_{1, k}, y_{m}\right): m \in M\right\}$.

\section{Algorithm 1}

(1) Set $V=\left\{(0,0), \mathrm{SE}_{1}, \mathrm{NW}_{1},\left(T M_{1, k}, T M_{2, k}\right)\right\}$ and $A=\left\{\left((0,0), \mathrm{SE}_{1}\right),\left((0,0), \mathrm{NW}_{1}\right)\right\}$.

(2) Take vertex $(x, y) \in V \backslash\left\{\left(T M_{1, k}, T M_{2, k}\right)\right\}$ with zero outdegree. If $(x, y)=\mathrm{SE}_{m}$, then go to Step 3. If $(x, y)=\mathrm{NW}_{m}$, then go to Step 4. If set $И\left\{\left(T M_{1, k}, T M_{2, k}\right)\right\}$ has no vertex with zero outdegree, then STOP.

(3) Draw a diagonal line starting from vertex $\mathrm{SE}_{m}$ until either east boundary $\left[\left(T M_{1, k}, 0\right),\left(T M_{1, k}, T M_{2, k}\right)\right]$ of the rectangle $\mathrm{R}$ is reached in vertex $\left(T M_{1, k}, y_{m}\right)$ or open south boundary $\left(\mathrm{SW}_{h}, \mathrm{SE}_{h}\right)$ of the rectangle $R_{h}, m+1 \leqslant h \leqslant k$, is reached. In the former case, set $V:=V \cup\left\{\left(T M_{1, k}, y_{m}\right)\right\}$ and $A:=A \cup\left\{\left(\mathrm{SE}_{m},\left(T M_{1, k}, y_{m}\right)\right),\left(\left(T M_{1, k}, y_{m}\right),\left(T M_{1, k}, T M_{2, k}\right)\right)\right\}$. In the latter case, set $V:=V \cup\left\{\mathrm{SE}_{h}, \quad \mathrm{NW}_{h}\right\}$, $A:=A \cup\left\{\left(\mathrm{SE}_{m}, \mathrm{SE}_{h}\right),\left(\mathrm{SE}_{m}, \mathrm{NW}_{h}\right)\right\}$. Go to Step 2 .

(4) Draw a diagonal line starting from vertex $\mathrm{NW}_{m}$ until either north boundary $\left[\left(0, T M_{2, k}\right),\left(T M_{1, k}, T M_{2, k}\right)\right]$ of the rectangle $R$ is reached in vertex $\left(x_{m}, T M_{2, k}\right)$ or open west boundary $\left(\mathrm{SW}_{h}, \mathrm{NW}_{h}\right)$ of the rectangle $R_{h}, m+1 \leqslant h \leqslant k$, is reached. In the former case, set $V:=V \cup\left\{\left(x_{m}, T M_{2, k}\right)\right\}$ and $A:=A \cup\left\{\left(\mathrm{NW}_{m}, \quad\left(x_{m}, T M_{2, k}\right)\right), \quad\left(\left(x_{m}, T M_{2, k}\right)\right.\right.$, $\left.\left.\left(T M_{1, k}, T M_{2, k}\right)\right)\right\}$. In the latter case, set $V:=V \cup\left\{\mathrm{SE}_{h}\right.$, $\left.\mathrm{NW}_{h}\right\}, A:=A \cup\left\{\left(\mathrm{NW}_{m}, \mathrm{SE}_{h}\right),\left(\mathrm{NW}_{m}, \mathrm{NW}_{h}\right)\right\}$. Go to Step 2.

In order to find the optimal trajectory (ie, optimal schedule) for the problem $F k|n=2| \Phi$, one can use the following algorithm.

\section{Algorithm 2}

(1) Construct the digraph $(V, A)$ using Algorithm 1 and find all its border vertices, that is, the vertices $(x, y)$ either of the form $\left(x_{m}, T M_{2, k}\right)$ or of the form $\left(T M_{1, k}, y_{m}\right)$.

(2) Construct the set of trajectories corresponding to the shortest paths in the digraph $(V, A)$ from the vertex $(0,0)$ to each of the border vertices.

(3) Find a trajectory in the set constructed at Step 2 that represents a schedule with minimal value of the objective function $\Phi$.

Algorithm 2 takes $O(k \log k)$ time (see Sotskov $\left.{ }^{28,29}\right)$. In the next section, we show how to generalize geometrical approach for the problem $F k\left|n=2, a_{j, m} \leqslant t_{j, m} \leqslant b_{j, m}\right| \Phi$.

\section{Problem Fk $\left|\mathbf{n}=\mathbf{2}, \mathbf{a}_{\mathbf{j}, \mathbf{m}} \leqslant \mathbf{t}_{\mathbf{j}, \mathbf{m}} \leqslant \mathbf{b}_{\mathbf{j}, \mathbf{m}}\right| \Phi$}

Let $\left(V_{t}, A_{t}\right)$ denote digraph $(V, A)$ constructed by Algorithm 1 for the problem $F k|n=2| \Phi$ with vector $t \in T$ of processing times. Let $\boldsymbol{\Lambda}_{t}$ be the set of all paths from vertex $(0,0)$ to vertex $\left(T M_{1, k}, T M_{2, k}\right)$ in the digraph $\left(V_{t}, A_{t}\right), t \in T$. As follows from Algorithm 1, the same path may belong to sets $\boldsymbol{\Lambda}_{t}$ constructed for different vectors $t \in T$ of processing times (recall that for any vector $t \in T$, we have $V_{t} \subseteq V^{0}$ ). Let $\Lambda=\mathbf{U}_{t \in T} \Lambda_{t}$. Notation $\lambda_{u}(t)$ will be used for a trajectory in rectangle $R$ defined by path $\lambda_{u} \in \Lambda_{t}$. Contrary to a path $\lambda_{u} \in \Lambda_{t}$, trajectory $\lambda_{u}(t)$ may be defined only when vector $t$ of processing times is fixed. Definition 1 given for permutation schedules $\pi_{\mathrm{r}}(t)$, defined by sequences $\pi_{\mathrm{r}} \in \mathbf{S}$, may be rewritten for trajectories (active schedules) defined by paths as follows: A set of paths $\boldsymbol{\Lambda}^{*} \subseteq \boldsymbol{\Lambda}$ is called a solution to the problem $F k\left|n=2, a_{j, m} \leqslant t_{j, m} \leqslant b_{j, m}\right| \Phi$ if for each feasible vector $t \in T$ of processing times the set $\boldsymbol{\Lambda}^{*}$ contains at least one path $\lambda_{r}$ such 
that trajectory $\lambda_{r}(t)$ is optimal. Let $m$ and $m^{*}$ be integers such that $m \geqslant 1$ and $m+m^{*} \leqslant k$.

Lemma 3 For any feasible vector $t \in T$ at step 3 (at step 4) of Algorithm 1, the diagonal line with origin in vertex $S E_{m}$ (in vertex $N W_{m}$ ) cannot intersect open south (west) boundaries of rectangles $R_{m+1}, R_{m+2}, \ldots, R_{m+m^{*-1}}$, if and only if the following $m^{*}$ inequalities hold:

$$
\begin{gathered}
\sum_{i=m}^{m+w-1} a_{2, i} \geqslant \sum_{i=m}^{m+w-1} b_{1, i+1}, w=1,2, \ldots, m^{*} \\
\left(\sum_{i=m}^{m+w-1} a_{1, i} \geqslant \sum_{i=m}^{m+w-1} b_{2, i+1}, w=1,2, \ldots, m^{*}\right)
\end{gathered}
$$

Proof First, we consider the case $m^{*}=1$. For any vector $t \in T$, trajectory of the form $\lambda_{u}(t)=\left[(0,0), \ldots, \mathrm{SE}_{\mathrm{m}}, \ldots\right.$, $\left.\left(T M_{1, k}, T M_{2, k}\right)\right], \quad \lambda_{u} \in \boldsymbol{\Lambda}_{t}$, cannot intersect open south boundary $\left(\mathrm{SW}_{m+1}, \mathrm{SE}_{m+1}\right)$ of rectangle $R_{m+1}$ if and only if

$$
a_{2, m} \geqslant b_{1, m+1}
$$

Indeed, the diagonal segment of trajectory $\lambda_{u}(t)$ with origin in vertex $\mathrm{SE}_{m}$ intersects open south boundary of rectangle $R_{m+1}$ only if $t_{2, m}<t_{1, m+1}$. However, from (28) it follows that $t_{2, m} \geqslant a_{2, m} \geqslant b_{1, m+1} \geqslant t_{1, m+1}$. On the other hand, if Inequality (28) does not hold, then there exists a vector $t \in T$ such that the diagonal segment of trajectory $\lambda_{u}(t)$ with origin in vertex $\mathrm{SE}_{m}$ intersects open south boundary of rectangle $R_{m+1}$. For the case $m^{*} \in\{2,3, \ldots, k-m\}$, we can repeat a similar argument $m^{*}$ times. As a result, Lemma 3 will be proven for step 3 of Algorithm 1 and vertex $\mathrm{SE}_{m}$. Owing to symmetry of jobs 1 and 2, Lemma 3 can be similarly proven for step 4 of Algorithm 1 and vertex $\mathrm{NW}_{\mathrm{m}}$.

Let $V^{*}=\mathbf{U}_{t \in T} V_{t}$ and $A^{*}=\mathbf{U}_{t \in T} A_{t}$. Owing to Lemma 3, we can restrict the number of vertices from set $V^{0}$ which have to be included in the set $V^{*}$. To restrict the number of arcs in the set $A^{*}$, we can use the following Lemmas 4 and $4^{*}$ where it is assumed that $b_{1, k+1}=T M_{1, k}$ and $b_{2, k+1}=T M_{2, k}$. Let $m$, $m^{*}$ and $h$ be integers such that $m \geqslant 1$ and $h=m+m^{*} \leqslant k+1$.

Lemma 4 For $h \leqslant k$, inclusions $\left(\mathrm{SE}_{m}, \mathrm{SE}_{h}\right) \in A^{*}$ and $\left(\mathrm{SE}_{m}\right.$, $\left.\mathrm{NW}_{h}\right) \in A^{*}\left(\right.$ for $h=k+1$, inclusions $\left(\mathrm{SE}_{m},\left(T M_{1, k}, y_{m}\right)\right) \in A^{*}$ and $\left.\left(\left(T M_{1, k}, y_{m}\right),\left(T M_{1, k}, T M_{2, k}\right)\right) \in A^{*}\right)$ hold if and only if the following system of linear inequalities has a solution:

$$
\begin{gathered}
\sum_{i=m}^{m+w-1} t_{2, i} \geqslant \sum_{i=m}^{m+w-1} t_{1, i+1}, w=1,2, \ldots, m^{*} \\
\sum_{i=m}^{m+w} t_{2, i}<\sum_{i=m}^{m+w-1} t_{1, i+1}+b_{1, h} \\
a_{i, j} \leqslant t_{i, j} \leqslant b_{i, j}, \quad i=1,2 ; j=m, m+1, \ldots, h
\end{gathered}
$$

Proof From Lemma 3, it follows that Inequalities (29) and (31) are necessary and sufficient for the existing vector $t \in T$ for which the diagonal line with origin in vertex $\mathrm{SE}_{m}$ does not intersect open south boundary of rectangles $R_{m+1}$, $R_{m+2}, \ldots, R_{h-1}$. Let $T^{*}$ denote the set of all vectors $t \in T$ where its components satisfy Inequalities (29) and (31). For $h \leqslant k$, Inequality (30) is necessary and sufficient for the existing vector $t^{*} \in T^{*}$ for which diagonal line with origin in vertex $\mathrm{SE}_{m}$ intersects open south boundary $\left(\mathrm{SW}_{h}, \mathrm{SE}_{h}\right)$ of rectangle $R_{h}$. (In Figure 1, all such diagonal lines are located in the black region.) Therefore for $h \leqslant k$, arcs $\left(\mathrm{SE}_{m}, \mathrm{SE}_{h}\right)$ and $\left(\mathrm{SE}_{m}, \mathrm{NW}_{h}\right)$ have to be included to the set $A_{t^{*}}$ at step 3 of Algorithm 1 if and only if system (29)-(31) has a solution.

We can prove Lemma 4 for the case $h=k+1$ similarly as for the case $h \leqslant k$. Note that for $h=k+1$, Inequality (30) definitely hold due to the assumption of $b_{1, k+1}=T M_{1, k}$.

Owing to symmetry between jobs 1 and 2 , the claim analogous to Lemma 4 (we call it Lemma $4^{*}$ ) can be proven for arcs $\left(\mathrm{NW}_{m}, \mathrm{SE}_{h}\right)$ and $\left(\mathrm{NW}_{m}, \mathrm{NW}_{h}\right)$ with $m \geqslant 1$ and $h=m+m^{*} \leqslant k$, and for arcs $\left(\mathrm{NW}_{m},\left(x_{m}, T M_{2, k}\right)\right)$ and $\left(\left(x_{m}\right.\right.$, $\left.\left.T M_{2, k}\right),\left(T M_{1, k}, T M_{2, k}\right)\right)$ with $m \geqslant 1$ and $h=k+1$. In particular, analogous system $\left(29^{*}\right)-\left(31^{*}\right)$ of linear inequalities used in Lemma $4^{*}$ instead of (29)-(31) is obtained from system (29)-(31) by replacing $t_{2, i}$ by $t_{1, i}, t_{1, i+1}$ by $t_{2, i+1}$, and $b_{1, h}$ by $b_{2, h}$. Using Lemmas 3, 4 and $4^{*}$ one can construct the digraph $\left(V^{*}, A^{*}\right)$ containing path set $\Lambda^{*}$ which is a solution to the problem $F k\left|n=2, a_{j, m} \leqslant t_{j, m} \leqslant b_{j, m}\right| \Phi$ for any regular criterion $\Phi$. To this end, we can use Algorithm 3 where $R_{k+1}$ denotes $R$.

\section{Algorithm 3}

(1) Set $V^{*}=\left\{(0,0), \mathrm{SE}_{1}, \mathrm{NW}_{1},\left(T M_{1, k}, T M_{2, k}\right)\right\}$ and $A^{*}=\left\{\left((0,0), \mathrm{SE}_{1}\right),\left((0,0), \mathrm{NW}_{1}\right)\right\}$.

(2) Take vertex $(x, y) \in V^{*} \backslash\left\{\left(T M_{1, k}, T M_{2, k}\right)\right\}$ with zero outdegree. If $(x, y)=\mathrm{SE}_{m}$, go to Step 3. If $(x, y)=\mathrm{NW}_{m}$, go to Step 4. If set $V^{*} \backslash\left\{\left(T M_{1, k}, T M_{2, k}\right)\right\}$ has no vertex with zero outdegree, go to Step 5.

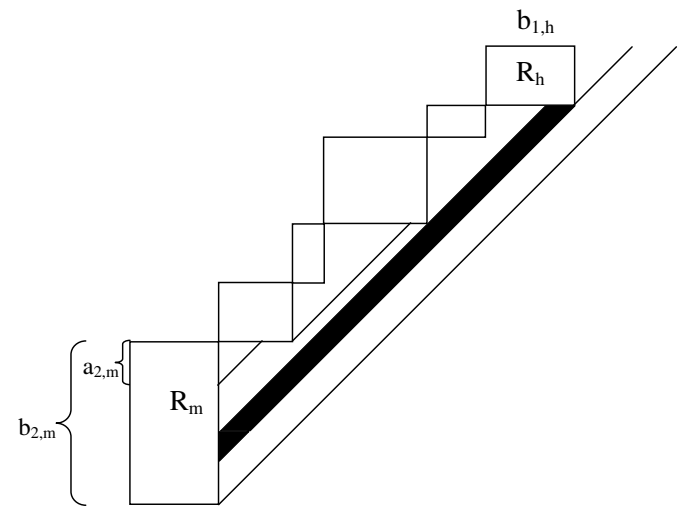

Figure 1 The case of Lemma 4 for $h-m=5$. 
(3) Test Inequalities (26) from Lemma 3 and define rectangle $R_{h}$ with minimal $h$ where its boundary may be reached by the diagonal line with origin in vertex $\mathrm{SE}_{m}$. If $h \leqslant k$, set $V^{*}:=V^{*} \cup\left\{\mathrm{SE}_{h}, \mathrm{NW}_{h}\right\}$ and $A^{*}:=A^{*} \cup\left\{\left(\mathrm{SE}_{m}\right.\right.$, $\left.\left.\mathrm{SE}_{h}\right),\left(\mathrm{SE}_{m}, \mathrm{NW}_{h}\right)\right\}$. If $h=k+1$, set $V^{*}:=V^{*} \cup\left\{\left(T M_{1, k}\right.\right.$, $\left.\left.y_{m}\right)\right\}, A^{*}:=A^{*} \cup\left\{\left(\mathrm{SE}_{m},\left(T M_{1, \mathrm{k}}, y_{m}\right)\right),\left(\left(T M_{1, k}, y_{m}\right),\left(T M_{1, k}\right.\right.\right.$, $\left.\left.\left.T M_{2, k}\right)\right)\right\}$. Go to Step 2.

(4) Test Inequalities (27) from Lemma 3 and define rectangle $R_{h}$ with minimal $h$ where its boundary may be reached by the diagonal line with origin in vertex $\mathrm{NW}_{m}$. If $h \leqslant k$, set $V^{*}:=V^{*} \cup\left\{\mathrm{SE}_{h}, \mathrm{NW}_{h}\right\}$ and $A^{*}:=A^{*} \cup\left\{\left(\mathrm{NW}_{m}\right.\right.$, $\left.\left.\mathrm{SE}_{h}\right),\left(\mathrm{NW}_{m}, \mathrm{NW}_{h}\right)\right\}$. If $h=k+1$, set $V^{*}:=V^{*} \cup\left\{\left(x_{m}\right.\right.$, $\left.\left.T M_{2, k}\right)\right\}, A^{*}:=A^{*} \cup\left\{\left(\mathrm{NW}_{m},\left(x_{m}, T M_{2, k}\right)\right),\left(\left(x_{m}, T M_{2, k}\right)\right.\right.$, $\left.\left.\left(T M_{1, k}, T M_{2, k}\right)\right)\right\}$. Go to Step 2.

(5) Solve system (29)-(31) for each vertices $\mathrm{SE}_{m} \in V^{*}$ and $\mathrm{SE}_{h} \in V^{*}$ such that $\left(\mathrm{SE}_{m}, \mathrm{SE}_{h}\right) \notin \mathrm{A}^{*}, 1 \leqslant m<h \leqslant k+1$. If system (29)-(31) has a solution and $h \leqslant k(h=k+1)$, then set $A^{*}:=A^{*} \cup\left\{\left(\mathrm{SE}_{m}, \mathrm{SE}_{h}\right),\left(\mathrm{SE}_{m}, \mathrm{NW}_{h}\right)\right\}$ (set $A^{*}:=A^{*} \cup\left\{\left(\mathrm{SE}_{m}\right.\right.$, $\left.\left.\left(x_{m}, \mathrm{TM}_{2, k}\right)\right)\right\}$ ).

(6) Solve system (29*)-(31*) for each vertices $\mathrm{NW}_{m} \in V^{*}$ and $\quad \mathrm{NW}_{h} \in V^{*} \quad$ such that $\left(\mathrm{NW}_{m}, \quad \mathrm{NW}_{h}\right) \notin A^{*}$, $1 \leqslant m<h \leqslant k+1$. If system $\left(29^{*}\right)-\left(31^{*}\right)$ has a solution and $h \leqslant k(h=k+1)$, then set $A^{*}:=A^{*} \cup\left\{\left(\mathrm{NW}_{m}, \mathrm{NW}_{h}\right),\left(\mathrm{NW}_{m}\right.\right.$, $\left.\left.\mathrm{SE}_{h}\right)\right\}$ (set $\left.A^{*}:=A^{*} \cup\left\{\left(\mathrm{NW}_{m},\left(T M_{1, k}, y_{m}\right)\right)\right\}\right)$. STOP.

In Figure 2, the digraph $\left(V^{*}, A^{*}\right)$ constructed by Algorithm 3 is shown for the example of the problem $\mathrm{F} 5\left|n=2, a_{j, m} \leqslant t_{j, m} \leqslant b_{j, m}\right| \Phi$ with lower and upper bounds of processing times given in Table 1 . This digraph represents the set of paths $\boldsymbol{\Lambda}^{*}$ (solution to the problem $\left.\mathrm{F} 5\left|n=2, a_{j, m} \leqslant t_{j, m} \leqslant b_{j, m}\right| \Phi\right)$ in the condensed form.

It is clear that Algorithm 3 takes polynomial time. Indeed, for each of vertices $V^{*}$ we have to test at most $k$ Inequalities (26) at step 3, and at most $k$ Inequalities (27) at step 4. At step 5 (step 6), for each pair of non-adjacent vertices in the constructed digraph $\left(V^{*}, A^{*}\right)$ we have to solve system
Table 1 Lower and upper bounds of processing times

\begin{tabular}{lrrrrr}
\hline Machine $m$ & $m=1$ & $m=2$ & $m=3$ & $m=4$ & $m=5$ \\
\hline$a_{1, m}$ & 2 & 19 & 8 & 4 & 12 \\
$b_{1, m}$ & 2 & 20 & 10 & 10 & 14 \\
$a_{2, m}$ & 16 & 3 & 4 & 2 & 4 \\
$b_{2, m}$ & 17 & 4 & 6 & 3 & 6 \\
\hline
\end{tabular}

(29)-(31) (system $\left(29^{*}\right)-\left(31^{*}\right)$, respectively) of at most $5 \mathrm{k}$ linear inequalities with at most $2 \mathrm{k}$ variables.

Lemmas 3, 4 and $4^{*}$ are valid for any regular criterion $\Phi$. Next, we show how to further minimize the set $\Lambda^{*}$ for the total completion time.

Problem $F k\left|n=2, \mathbf{a}_{\mathbf{j}, \mathbf{m}} \leqslant \mathbf{t}_{\mathbf{j}, \mathbf{m}} \leqslant \mathbf{b}_{\mathbf{j}, \mathbf{m}}\right| \mathbf{\Sigma} \mathbf{C}_{\mathbf{i}, \mathbf{k}}$

Let $D\left(\lambda_{r}(t)\right)$ denote the sum of the lengths of the all horizontal and vertical segments in the part of trajectory $\lambda_{r}(t)=\left[(0,0), \ldots,(x, y),\left(T M_{1, k}, T M_{2, k}\right)\right]$ from vertex $(0,0)$ to the border vertex $(x, y)$. Next, we show that the total completion time for the schedule presented by trajectory $\lambda_{r}(t)$ (for brevity, $\operatorname{TCT}\left(\lambda_{r}(t)\right)$ ) can be calculated as follows:

$$
\operatorname{TCT}\left(\lambda_{r}(t)\right)=T M_{1, k}+T M_{2, k}+D\left(\lambda_{r}(t)\right)
$$

If $D\left(\lambda_{r}(t)\right)=0, \quad$ then $\quad \mathrm{TCT}\left(\lambda_{r}(t)\right)=T M_{1, k}+T M_{2, k}$. If $D\left(\lambda_{r}(t)\right)>0$, then each vertical (horizontal) segment of trajectory $\lambda_{r}(t)$ from vertex $(0,0)$ to the border vertex $(x$, $y$ ) adds to the sum $T M_{1, k}+T M_{2, k}$ the value equal to the length of this segment since exactly one job waits in each time point corresponding to the vertical (horizontal) segment of trajectory $\lambda_{r}(t)$. The length of the segment $\left[(x, y),\left(T M_{1, k}\right.\right.$, $\left.T M_{2, k}\right)$ ] is not added to the sum $D\left(\lambda_{r}(t)\right)$ since no job waits in each time point corresponding to this segment (one job is already completed).

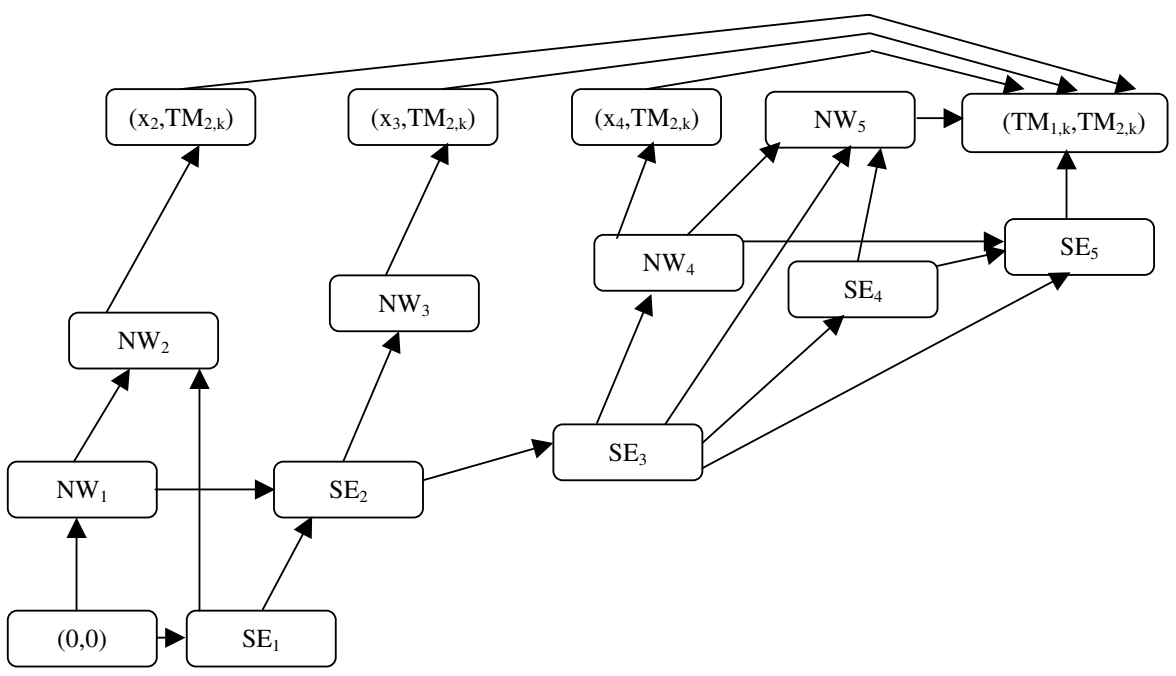

Figure 2 Digraph $\left(V^{*}, A^{*}\right)$ constructed by Algorithm 3. 
From Equality (32), it follows that minimizing total completion time is equivalent to minimizing the value of $D\left(\lambda_{r}(t)\right)$. For any path $\lambda_{w} \in \boldsymbol{\Lambda}$, we can define vector $t^{w}=$ $\left(t_{1,1}^{w}, t_{1,2}^{w}, \ldots, t_{1, k}^{w}, t_{2,1}^{w}, t_{2,2}^{w}, \ldots, t_{2, k}^{w}\right) \in T$ as follows. If point $\mathrm{SE}_{m}$ or point $\mathrm{SW}_{m}$ belongs to the horizontal segment of trajectory $\lambda_{w}(t)$, we set $t_{1, m}^{w}=b_{1, m}$, otherwise we set $t_{1, m}^{w}=a_{1, m}$. If point $\mathrm{NW}_{m}$ or point $\mathrm{SW}_{m}$ belongs to the vertical segment of trajectory $\lambda_{w}(t)$, we set $t_{2, m}^{w}=b_{2, m}$, otherwise we set $t_{2, m}^{w}=a_{2, m}$. Let notation $\left(\mathrm{SE}_{m}, \mathrm{SE}_{h}\right) \in \lambda_{w}$ mean that path $\lambda_{w}$ includes arc $\left(\mathrm{SE}_{m}, \mathrm{SE}_{h}\right)$. Let $\mathrm{SE}_{k+1}$ denote vertex $\left(T M_{1, k}\right.$, $\left.y_{m}\right)$ and $\mathrm{NW}_{k+1}$ denote vertex $\left(x_{m}, T M_{2, k}\right)$.

Theorem 3 The set $\left\{\lambda_{w}\right\}$ is a solution to the problem $F k\left|n=2, a_{j, m} \leqslant t_{j, m} \leqslant b_{j, m}\right| \Sigma C_{i, k}$ if and only if the following three conditions are satisfied:

(i) If either $\left(\mathrm{SE}_{m}, \mathrm{SE}_{h}\right) \in \lambda_{w}$ or $\left(\mathrm{SE}_{m}, \mathrm{NW}_{h}\right) \in \lambda_{w}$, then Inequalities (26) hold along with

$$
\sum_{i=m}^{h-1} b_{2, i}<\sum_{i=m}^{h-1} a_{1, i+1}+b_{1, h}
$$

(ii) If either $\left(\mathrm{NW}_{m}, \mathrm{SE}_{h}\right) \in \lambda_{w}$ or $\left(\mathrm{NW}_{m}, \mathrm{NW}_{h}\right) \in \lambda_{w}$, then Inequalities (27) hold along with

$$
\sum_{i=m}^{h-1} b_{1, i}<\sum_{i=m}^{h-1} a_{2, i+1}+b_{2, h}
$$

(iii) Schedule represented by trajectory $\lambda_{w}\left(t^{w}\right)$ is optimal for the problem $F k|n=2| \sum C_{i, k}$ with vector $t^{w}$ of processing times.

Proof Sufficiency. First, we show that from conditions (i) and (ii) it follows that path $\lambda_{w}$ is contained in the digraph $\left(V_{t}, A_{t}\right)$ constructed by Algorithm 1 for any feasible vector $t \in T$. We assume that $\left(\mathrm{SE}_{m}, \mathrm{SE}_{h}\right) \in \lambda_{w}$. From condition (i), it follows that Inequalities (26) and (33) hold. Thus, system of linear Inequalities (29)-(30) is satisfied for any vector $t \in T$. Therefore, due to Lemma 3 , set $A_{t}$ contains arc $\left(\mathrm{SE}_{m}, \mathrm{SE}_{h}\right)$ for any vector $t \in T$. Similarly if $\left(\mathrm{NW}_{m}, \mathrm{SE}_{h}\right) \in \lambda_{w}$, then condition (ii) implies that Inequalities (27) and (34) hold. Therefore, set $A_{t}$ contains arc $\left(\mathrm{NW}_{m}, \mathrm{SE}_{h}\right)$ for any vector $t \in T$. Arguing in a similar way for each arc in the path $\lambda_{w}$, we conclude that the whole path $\lambda_{w}$ is contained in the digraph $\left(V_{t}, A_{t}\right)$ for any vector $t \in T$. From condition (iii) we get inequality $\operatorname{TCT}\left(\lambda_{r}\left(t^{w}\right)\right)-\operatorname{TCT}\left(\lambda_{w}\left(t^{w}\right)\right) \geqslant 0$ for each trajectory $\lambda_{r}\left(t^{w}\right)$ with path $\lambda_{r}$ from the digraph $\left(V_{t^{w}}, A_{t^{w}}\right)$. Therefore due to (32), we get

$$
D\left(\lambda_{r}\left(t^{w}\right)\right)-D\left(\lambda_{w}\left(t^{w}\right)\right) \geqslant 0
$$

We consider arbitrary feasible vector $t=\left(t_{1,1}, t_{1,2}, \ldots, t_{1, \mathrm{k}}\right.$, $\left.t_{2,1}, t_{2,2}, \ldots, t_{2, \mathrm{k}}\right) \in T$ of the processing times. Since path $\lambda_{w}$ is contained in the digraph $\left(V_{t}, A_{t}\right)$, replacing $t_{j, m}^{w}=a_{j, m}$ by $t_{j, m} \geqslant a_{j, m}$ cannot change the value $D\left(\lambda_{w}\left(t^{w}\right)\right)$ but can increase the value $D\left(\lambda_{r}\left(t^{w}\right)\right)$. Due to the same reason, after replacing $t_{j, m}^{w}=b_{j, m}$ by $t_{j, m}<b_{j, m}$ the value $D\left(\lambda_{w}\left(t^{w}\right)\right)$ is decreased by the maximal possible value, namely, by value $b_{j, m}-t_{j, m}$. So, taking into account (35), we get $\operatorname{TCT}\left(\lambda_{r}(t)\right)$ $-\mathrm{TCT}\left(\lambda_{w}(t)\right)=D\left(\lambda_{r}(t)\right)-D\left(\lambda_{w}(t)\right) \geqslant D\left(\lambda_{r}\left(t^{w}\right)\right)-D\left(\lambda_{w}\left(t^{w}\right)\right)$ $\geqslant 0$. To finish sufficiency proof, we have to compare trajectory $\lambda_{w}(t)$ with trajectory $\lambda_{u}(t)$ such that the digraph $\left(V_{t^{w}}, A_{t^{w}}\right)$ does not contain path $\lambda_{u}$. Let trajectory $\lambda_{u}\left(t^{w}\right)$ represent schedule with the same orders of jobs on all $k$ machines as the schedule represented by trajectory $\lambda_{u}(t)$. (Note that the former schedule may not be active.) From condition (iii), we get inequality $D\left(\lambda_{u}\left(t^{w}\right)\right)-D\left(\lambda_{w}\left(t^{w}\right)\right) \geqslant 0$. Arguing similarly as for trajectory $\lambda_{r}\left(t^{w}\right)$, we can show that $\operatorname{TCT}\left(\lambda_{u}(t)\right)-\operatorname{TCT}\left(\lambda_{w}(t)\right)=D\left(\lambda_{u}(t)\right)-D\left(\lambda_{w}(t)\right) \geqslant D\left(\lambda_{u}\left(t^{w}\right)\right)$ $-D\left(\lambda_{w}\left(t^{w}\right)\right) \geqslant 0$.

Necessity. If either condition (i) or condition (ii) is not satisfied, then due to Lemma 3 there exists feasible a vector $t \in T$ such that digraph $\left(V_{t}, A_{t}\right)$ does not contain path $\lambda_{w}$. Therefore, single-element set $\left\{\lambda_{w}\right\}$ cannot be a solution to the problem $F k\left|n=2, a_{j, m} \leqslant t_{j, m} \leqslant b_{j, m}\right| \Sigma C_{i, k}$. If schedule represented by trajectory $\lambda_{w}\left(t^{w}\right)$ is not optimal for the problem $F k|n=2| \Sigma C_{i, k}$ with vector $t^{w} \in T$ (violation of condition (iii)), then set $\left\{\lambda_{w}\right\}$ is not a solution to the problem $F k\left|n=2, a_{j, m} \leqslant t_{j, m} \leqslant b_{j, m}\right| \Sigma C_{i, k}$.

Testing whether a singleton is a solution to problem $F k\left|n=2, a_{j, m} \leqslant t_{j, m} \leqslant b_{j, m}\right| \Sigma C_{i, k}$ takes $O\left(k^{3}\right)$ time. Indeed, we can use Algorithm 2 for some fixed feasible vector $t$ of the processing times and construct all optimal trajectories for the problem $F k|n=2| \Sigma C_{i, k}$. Then we can test Theorem 3 for each of the constructed optimal trajectories. Number of optimal trajectories for the problem $F k|n=2| \Sigma C_{i, k}$ is restricted by the number of border vertices asymptotically restricted by $O(k)$. It is clear that testing Theorem 3 takes $O\left(k^{2}\right)$ time.

For the problem $F 5\left|n=2, a_{j, m} \leqslant t_{j, m} \leqslant b_{j, m}\right| \Sigma C_{i, 5}$ with bounds of processing times given in Table 1 , conditions of Theorem 3 hold for the path $\lambda_{w}=\left((0,0), \mathrm{SE}_{1}, \mathrm{SE}_{2}, \mathrm{NW}_{3}\right.$, $\left.\left(x_{3}, T M_{2,5}\right),\left(T M_{1,5}, T M_{2,5}\right)\right)$. Owing to Theorem 3, singleton $\left\{\lambda_{w}\right\}$ is a solution to the problem $F 5 \mid n=2, a_{j, m} \leqslant$ $t_{j, m} \leqslant b_{j, m} \mid \Sigma C_{i, 5}$.

\section{Conclusion}

Sufficient conditions are proven for fixing the order of two adjacent jobs in an optimal schedule for the problem $F k\left|a_{j, m} \leqslant t_{j, m} \leqslant b_{j, m}\right| \Sigma C_{i, k}$ with $n$ jobs and two or three machines. Such conditions may be used to minimize solution $\mathbf{S}^{*}$ of problem $F k\left|a_{j, m} \leqslant t_{j, m} \leqslant b_{j, m}\right| \Sigma C_{i, k}$ with $k \leqslant 3$. It is clear that Theorems 2 and 3 can be generalized for $k>3$. In the previous section, necessary and sufficient conditions are proven for the existing single-element solution to the problem $F k\left|n=2, a_{j, m} \leqslant t_{j, m} \leqslant b_{j, m}\right| \Sigma C_{i, k}$. This result can be 
generalized for a jobshop problem with two jobs and other regular criterion. In this case, we need to modify Equality (32) in an appropriate way. Note that all conditions presented in Theorems $1-3$ can be tested in polynomial time.

Acknowledgments - The research of the first author was partially supported by INTAS (project 00-217). The research of the second author was supported by Kuwait University Research Administration (project EM 01/01). The research of the third author was supported by NSC of Taiwan under projects NSC 89-2416-H002-039 and NSC 912416-H-002-006

\section{References}

1 Gonzalez T and Sahni S (1978). Flow shop and job shop schedules. Ops Res 26: 36-52.

2 Della Croce F, Narayan V and Tadei R (1996). The twomachine total completion time flow shop problem. Eur $J \mathrm{Opl}$ Res 90: 227-237.

3 Allahverdi A (2000). Minimizing mean flowtime in a twomachine flowshop with sequence independent setup times. Comput Ops Res 27: 111-127.

4 Rajendran C and Ziegler H (1997). An efficient heuristic for scheduling in a flowshop to minimize total weighted flowtime of jobs. Eur J Opl Res 103: 129-138.

5 Woo HS and Yim DS (1998). A heuristic algorithm for mean flowtime objective in flowshop scheduling. Comput Ops Res $\mathbf{2 5}$ : 175-182.

6 Allahverdi A and Aldowaisan T (2002). New heuristics to minimize total completion time in m-machine flowshops. Int $J$ Product Econ 77: 71-83.

7 Kamburowski J (1999). Stochastically minimizing the makespan in two-machine flow shops without blocking. Eur J Opl Res 112: 304-309.

8 Elmaghraby S and Thoney KA (2000). Two-machine flowshop problem with arbitrary processing time distributions. IIE Trans 31: 467-477.

9 Allahverdi A (1999). Stochastically minimizing total flowtime in flowshops with no waiting space. Eur J Opl Res 113: 101-112.

10 Allahverdi A and Mittenthal J (1994). Two-machine ordered flowshop scheduling under random breakdowns. Math Comput Modelling 20: 9-17.

11 Lawler EL, Lenstra JK, Rinnooy Kan AHG and Shmoys DB (1993). Sequencing and scheduling: algorithms and complexity. In: Graves SS, Rinnooy Kan AHG and Zipkin P (eds). Handbooks in Operations Research and Management Science, 4: Logistics of Production and Inventory. North-Holland, New York, pp 445-522.
12 Tanaev VS, Sotskov YN and Strusevich VA (1994). Scheduling Theory: Multi-Stage Systems. Kluwer Academic Publishers: The Netherlands, Dordrecht.

13 Ishii H, Masuda T and Nishida T (1987). Two machine mixed shop scheduling problems with controllable machine speeds. Discrete Appl Math 17: 29-38.

14 Janiak A (1988). General flow-shop scheduling with resource constraints. Int J Product Res 26: 125-138.

15 Strusevich VA (1995). Two machine flow shop scheduling problem with no wait in process: Controllable machine speeds. Discrete Appl Math 59: 75-86.

16 Lei L and Wang TJ (1991). The minimum common-cycle algorithm for cycle scheduling of two hoists with time window constraints. Mngt Sci 37: 1629-1639.

17 Chen H, Chu C and Proth JM (1998). Cyclic scheduling of a hoist with time window constraints. IEEE Trans Robotics Automat 14: 144-152.

18 Lai TC, Sotskov YN, Sotskova NY and Werner F (1997). Optimal makespan scheduling with given bounds of processing times. Math Comput Modelling 26: 67-86.

19 Lai TC and Sotskov YN (1999). Sequencing with uncertain numerical data for makespan minimization. J Opl Res Soc 50: 230-243.

20 Lai TC, Sotskov YN, Sotskova NY and Werner F. Mean flow time minimization with given bounds of processing times. Eur $J$ Opl Res., in press.

21 Allahverdi A and Sotskov YN (2003). Two-machine flowshop minimum-length scheduling problem with random and bounded processing times. Int Trans Opl Res 10: 65-76.

$22 \mathrm{Ku}$ PS and Niu SC (1986). On Johnson's two-machine flow shop with random processing times. Ops Res 34: 130-136.

23 Pinedo M (1995). Scheduling: Theory, Algorithms, and Systems. Prentice-Hall: Englewood Cliffs, NJ.

24 Akers SB and Friedman J (1955). A non-numerical approach to production scheduling problems. Ops Res 3: 429-442.

25 Szwarc W (1960). Solution of the Akers-Friedman scheduling problem. Ops Res 8: 782-788.

26 Hardgrave WW and Nemhauser G (1963). A geometric model and graphical algorithm for a sequencing problem. Ops Res 11: 889-900.

27 Brucker $P$ (1988). An efficient algorithm for the job-shop problem with two jobs. Computing 40: 353-359.

28 Sotskov YN (1985). Optimal scheduling two jobs with regular criterion. Design Processes Automating: 86-95 (in Russian).

29 Sotskov YN (1991). The complexity of shop-scheduling problems with two or three jobs. Eur J Opl Res 53: 326-336.

Received April 2003; accepted November 2003 after one revision 